\title{
Estilos de gestión y extensión en predios ganaderos: Un estudio de caso en Uruguay
}

\section{Extension and Management styles cattle farms: a case study in Uruguay}

\author{
Carlos Molina $^{1,2^{*}}$ (I) , Jorge Alvarez ${ }^{1}$ (D), Pedro de Hegedus ${ }^{1}$ (iD)
}

\section{RESUMEN}

Para incrementar el conocimiento de factores determinantes de resultados productivos y económicos a escala de predio criador familiar; se profundizó en comprender mejor el qué y cómo hacen los productores lo que hacen y los motivos que tienen para hacerlo. Se analizaron características del sistema de producción, el manejo de animales y de pasturas. Se profundizó en el conocimiento de cómo realizan la gestión del predio, la integración del equipo de gestión y el proceso de toma de decisiones. Se entrevistó a los responsables de dos predios ganaderos criadores familiares de los departamentos de Rocha y Cerro Largo; que logran resultados productivos y económicos diferentes. Las diferencias en la forma de gestionar los establecimientos se representan como dos estilos de gestión distintos. El primer estilo es definido como "analítico-pastoril", donde las actividades de gestión tienen un lugar destacado dentro de las actividades prediales, con elevados niveles de intervención por parte del decisor en los procesos clave del sistema productivo. Se jerarquiza el papel del aporte de información técnica y del trabajo en grupos. El segundo estilo es definido como "experiente-ganadero", donde las actividades de gestión ocupan un papel secundario, la toma de decisiones se basa en la experiencia, y los niveles de intervención se identificaron como marginales en procesos productivos importantes. Resulta relevante que el primer estilo de gestión estuvo asociado al logro de elevados resultados prediales, tanto en lo productivo como en lo económico; en tanto que los resultados en el segundo indicaron un menor desempeño en ambas áreas.

Palabras claves: Ganaderos familiares, criadores, gestión, estilos, resultados.

\begin{abstract}
To have a better understanding of the factors that determine the production and economic results scale family breeder farm, was deepened knowledge and understanding of what and how producers do what they do and their reasons for doing so. To achieve this goal we analyzed the characteristics of the production system, in relation to the handling of animals and pasture. Also raised as a necessity as the knowledge of farm management performed, as is the integration of the management team and the decision-making process. To do this we interviewed two family farmers livestock producers departments Rocha and Cerro Largo, that production and economic results were different. The differences in the way they manage establishments are represented as two management styles for each case study. The first style is defined as "analytical-pastoral" where management activities are prominent in farm activities, with high levels of intervention by the decision maker in the key processes of the production system. Providing technical information and working in groups are key elements. The second style is defined as "experient-farmer", where management activities occupied a secondary role, decision are made based upon experience, and the levels of intervention were identified as marginal in most important production processes. It is relevant that the first management style was associated with achieving very good results on the farm, both productive and economically, while the results in the second indicated a lower performance in both areas.
\end{abstract}

Keywords: Livestock farmers, breeders, management, styles, results.

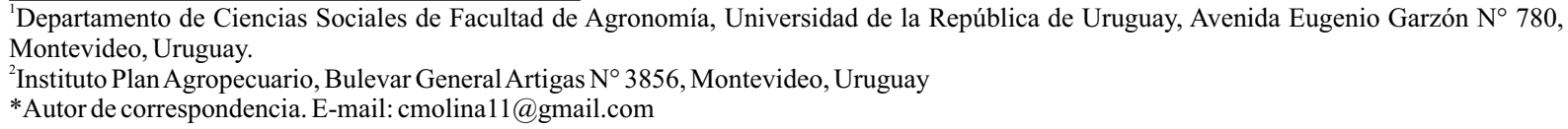




\section{INTRODUCCIÓN}

El agro uruguayo ha sufrido transformaciones importantes en las últimas dos décadas. Es un período de profundos cambios: la tierra se valorizó fuertemente (en siete veces), el número de vacunos ascendió a más de 11 millones, el stock ovino descendió a menos de 7 millones, se incrementó notablemente el área ocupada por agricultura y por forestación y el número de productores, principalmente familiares, descendió en más de 10000 .

En el período, la ganadería tuvo un importante aumento de productividad. Se intensificaron los procesos de recría-engorde pero en la cría, la mayor producción de terneros se da a través de mayor cantidad de hembras entoradas, y no por un aumento de productividad por vaca.

El objetivo general de esta investigación es contribuir en el conocimiento de factores determinantes de los resultados productivos-económicos a escala de predio criador familiar. Para el logro del objetivo general se plantearon los siguientes objetivos específicos:

1. Mejorar el conocimiento y la comprensión del qué y el cómo hacen los productores

2. Analizar características del sistema de producción, en lo relacionado con el manejo animales-pasturas.

3. Conocer cómo realizan la gestión predialtoma de decisiones.

4. Analizar el papel del asesoramiento técnico y la extensión rural (ATER)

El marco teórico bajo el cual se desarrolló el trabajo es el enfoque sistémico y específicamente y con una referencia teórica directa con éste, el enfoque global de la empresa agropecuaria con el objetivo de comprender-diagnosticar el funcionamiento de las mismas. El enfoque global de la empresa agropecuaria profundiza en el conocimiento de objetivos, finalidades, historia y estrategias de los predios. Es una metodología de comprensión del proceso de toma de decisiones a nivel predial que permite conocer los factores que lo afectan.

El abordaje metodológico empleado se ubica en el paradigma cualitativo. El procedimiento que se utilizó corresponde a un Estudio de Caso.

Los dos casos estudiados son predios familiares, de acuerdo a la definición del MGAP, con resultados diferentes tanto productivos como económicos. $\mathrm{La}$ ubicación de los predios es en los departamentos de Rocha y Cerro Largo. Se entrevistaron 4 personas, integrantes de los 2 predios familiares estudiados y 4 vecinos de los mismos.

\section{Marco conceptual: gestión en predios familiares}

Marshall et al. (1994) sostienen que desde la perspectiva del enfoque sistémico, para comprender los fenómenos complejos es necesario tomar en cuenta todos los rasgos de la complejidad real, sin desintegrarla. Postulan que estudiar la empresa como sistema es considerar en primer lugar el conjunto antes que profundizar el estudio de las partes, es poner el acento en las interacciones, y fuentes de complejidad, tanto en procesos técnicos como en la toma de decisiones del productor.

Aplicando este concepto a predios ganaderos, parece claro que para entender cualquier aspecto de los mismos, desde el sistema productivo hasta la gestión del mismo, es necesario entender su lógica de funcionamiento.

Osty (1978) acuñó el término «sistema familiaexplotación» que asocia explícitamente el estudio de las explotaciones agrarias con la metodología sistémica y aporta un matiz social a la definición de explotación. Este autor definió la explotación agraria como un todo organizado, cuyo funcionamiento y necesidades sólo pueden ser comprendidos teniendo en cuenta los objetivos y las opiniones de los productores. Este planteamiento posibilita una nueva forma de abordar el estudio de los sistemas de producción denominada «aproximación global a la explotación» (Bonneviale et al. ,1989). En el estudio del sistema familiaexplotación se tendrían en cuenta, además de las variables consideradas en el estudio de los sistemas de producción relacionadas con los recursos y las producciones de las explotaciones, variables relativas a aspectos sociales del ganadero y familia (edad-formación- 
participación de la familia-sucesión).

Bajo este concepto de sistema familia-explotación, las herramientas de gestión fijadas por el productor y las decisiones adoptadas no se determinan exclusivamente basándose en la situación económica-financiera de corto plazo, sino que en muchos casos abarca proyectos de un grupo familiar durante más de una generación. Desde esta perspectiva, Bonnevialle et al. (1989) ofrecen una representación conceptual simplificadora de la complejidad del sistema familia-explotación, descomponiéndola en tres subsistemas principales interrelacionados entre sí. Los subsistemas son: el relacionado con las operaciones (sistema de producción-operativo), el relacionado con las decisiones propiamente (sistema de decisión), y el relacionado con la información (sistema de información), a través del cual los anteriores se vinculan.

En el mismo sentido Marshall et al. (1994) sostienen que el funcionamiento del predio y la resolución de los problemas que se plantean en el mismo, se basan en dos niveles de acción: el sistema de decisión y el sistema de producción, interconectados entre sí a través del sistema de información.

Según Ruiz y Oregui (2001) el sistema de decisión es el entorno en el que, sobre la base de la información disponible referente al predio y al medio en el que el mismo se ubica, surgen las directrices de orden estratégico y táctico que rigen el sistema operador. En realidad se descompone a su vez en tres niveles de decisión: de finalidades, de dirección estratégica y de dirección táctica. Según Pedemonte (2014) el sistema de producción es el espacio donde tienen lugar todas las operaciones inherentes a la gestión de los procesos productivos. Se refiere a la combinación entre los distintos factores de producción, es decir, los elementos biológico, de trabajo y financiero, así como a las acciones encaminadas hacia la gestión de los procesos productivos.

De acuerdo a Ruiz y Oregui (2001) la interacción entre todos los elementos productivos del sistema se realiza por medio de técnicas y de prácticas. Las primeras constituyen un conjunto ordenado de operaciones que, basados en una serie de conocimientos de origen científico y/o empírico, o más frecuentemente aún, en ambos a la vez, persiguen la consecución de una finalidad. Por su parte las prácticas son el conjunto de actividades elementales que realiza el agricultor, su manera de hacer las cosas, acorde a su propia percepción del proceso productivo.

Siempre según Ruiz y Oregui (2001) mientras las primeras pueden ser descritas independientemente del agricultor o del ganadero pues están en el orden del conocimiento, las segundas, que están en el orden de la acción, requieren de ésta para su estudio. Por ello, el análisis de las prácticas puede ser revelador tanto del proyecto de explotación, como de su situación en un momento dado. En consecuencia, cualquier intento de estudio o representación del sistema técnico de una explotación debe hacerse en colaboración estrecha con los productores y debe reflejar sus opinionesrepresentaciones del sistema de producción.

El sistema de información funciona como nexo de unión entre los sistemas de decisión y de producción. Sostiene Ruíz y Oregui (2001) es uno de los lugares donde se establece la relación con el medio. La actividad de información consiste fundamentalmente en la traducción de los fenómenos observados a conceptos, por lo que depende de la formación, experiencia y ambiente del productor.

El proceso de toma de decisiones se basa en un conjunto de prácticas adoptadas por el ganadero. Las mismas están obviamente condicionados por la experiencia adquirida en el desempeño de la actividad (Beranger y Vissac, 1994).

El predio ganadero está dentro de un sistema social, con el cual interactúa y que influye en la toma de decisiones. Esta realidad debe ser considerada si se pretende caracterizar el sistema de gestión de un predio en particular. O sea que para gestionar todo el sistema es necesario operar en los subsistemas mencionados, que están interrelacionados de tal forma que se retroalimentan en su funcionamiento.

Respecto a la información de las decisiones según Alvarez (2005) existen diferentes modelos de repre- 
sentación de la toma de decisiones y del rol que juega la información en los mismos. Existen los modelos lineales -el proceso decisorio como una secuencia lineal de etapas- y los no lineales, donde no existe una continuidad entre el inicio y el fin de la toma de decisiones.

El enfoque global particularmente y como ya dijimos, se posiciona dentro del planteo de los modelos no lineales.

Según Alvarez (2005) la información es un insumo con características especiales, utilizado en el proceso de toma de decisiones. Es posible que a mejor información deberían seguir mejores decisiones; y a mejores decisiones, un mejor logro de objetivos y metas planteadas.

Según Figari (2008) uno de los rasgos considerados más distintivos del funcionamiento de productores familiares es la integración prácticamente total de las actividades productivas y domésticas, la utilización casi exclusiva de trabajo familiar, cuyo objetivo central es la reproducción de sus condiciones de vida y de trabajo. Sostiene Figari (2008) que a partir de la obra de Chayanov se identifica una tendencia dominante a concebir a los campesinos como parte de una economía específica y singular, que coexiste en el sistema económico capitalista.

Dice Figari (2008) que en este rasgo propio de la economía familiar se encuentra uno de los principales problemas del modelo de gestión desarrollado a partir de la economía clásica ya que, a diferencia de lo que ocurre en las empresas industriales, en los establecimientos agropecuarios en general, pero especialmente en los pequeños establecimientos familiares, la superposición del ámbito empresarial-familiar es un elemento estructural de este tipo de unidades económicas.

En Francia desarrollaron una propuesta metodológica concreta para realizar el estudio del funcionamiento de predios agropecuarios de tipo familiar y el asesoramiento técnico, desde otra perspectiva, denominada "Enfoque Global de la explotación Agrícola (EGEA)" según Figari (2008). La misma está basada en el enfo- que sistémico y bajo la premisa de que los productores tienen sus razones para hacer lo que hacen. Se trata en realidad de dos métodos: el primero observa y describe al sistema productivo en cuestión, el segundo analiza los problemas detectados, establece un diagnóstico y plantea posibles soluciones.

Según Figari (2008) estos nuevos enfoques sistémicos reposicionan al productor como sujeto de la acción y no como simple destinatario de tecnología moderna. La intervención técnica es vista como un "dialogo de saberes": una relación biunívoca entre "saberes" diferentes pero complementarios. Sobre esa base se establece una relación de horizontalidad y respeto. Se trabaja sobre motivaciones y objetivos reales de los productores. El productor que no aplica tecnología de avanzada no es visto como un "atrasado" o retrógrado que se resiste a la modernización, tal cual se desprende del paradigma difusionista de innovaciones de E. Rogers (caracterizado por la linealidad para entender el cambio técnico: unos investigan, otros transfieren, otros adoptan). El rol del técnico asesor es el de facilitar comprender al productor el funcionamiento del predio entendido como una totalidad que lo incluye, a los efectos de una mejor toma de decisiones (de Hegedus y Morales, 1996).

\section{MATERIALY MÉTODOS}

La estrategia seleccionada para esta investigación fue el estudio de caso. Estudio de Caso implica el análisis de pocos "casos" a los efectos de ganar conocimiento en profundidad de los mismos, en función de los intereses del investigador (Platt, 1992; Stoecker, 1991; Yin, 1989). Como diseño de investigación se caracteriza por su adaptabilidad al estudio de fenómenos contemporáneos en su contexto real. Esta característica es útil para el estudio de fenómenos complejos desde una perspectiva sistémica (Wilson y Morren, 1990).

Dos aspectos centrales de Estudio de Caso son: i) tener objetivos que guíen el trabajo operativo, y ii) tener un marco conceptual con el cual interpretar los resultados, y facilitar el análisis (en este trabajo es el enfoque 
sistémico). Esto facilita el procesamiento de la información que será cualitativo y permite ganar en riqueza de contenido; en contraposición resumir la información se transforma en una de sus mayores desventajas. Es un proceso creativo en el cuál se estudian las respuestas obtenidas -en función del marco conceptual establecido- buscando una lógica emergente que las vincule e integre (Patton, 1980). Es una herramienta valiosa de investigación en las ciencias sociales; procura entender a las personas involucradas en el fenómeno estudiado tal como ellas se manifiestan en la vida real, mientras que los métodos cuantitativos se centran en obtener información a través de encuestas (Yin, 1994). Además, en el método de estudio de caso los datos pueden ser obtenidos desde una variedad de fuentes, tanto cualitativas como cuantitativas. Los instrumentos para recabar la información fueron la observación directa, entrevistas abiertas y el análisis de datos prediales registrados en diversos soportes (libretas-almanaques-planillas electrónicas).

En función del diseño, es de casos-múltiple. Se trabajó con dos predios ganaderos criadores familiares, participantes del Programa de monitoreo de empresas ganaderas del Plan Agropecuario y se los comparó en aspectos estructurales (escala, subdivisiones), aspectos relacionados a prácticas productivas, de gestión, el papel del asesoramiento técnico y la extensión rural, y los resultados físicos-económicos logrados.

En las entrevistas a productores, la unidad de análisis es el productor y su familia. El estudio involucró dos productores familiares ganaderos criadores, localizados en los departamentos de Rocha y Cerro Largo. El tamaño de los predios variaba entre 195 y 240 ha.

La selección de los casos se realizó en función de las siguientes características: predio ganadero criador, mano de obra familiar principalmente, más del $70 \%$ del ingreso proveniente de la actividad ganadera, resultados productivos-económicos diferentes, integrantes del Programa de monitoreo de predios ganaderos del Plan Agropecuario.

Para la obtención de la información se realizaron entrevistas abiertas a los 2 productores y sus familias
(4 personas en total), observación directa a través de visitas a predio y análisis de registros físicos y económicos disponibles. Los registros físicos-económicos estaban disponibles a partir del uso por parte de los productores de una herramienta de registración denominada Carpeta Verde. A partir de la entrevista se relevaron aspectos relacionados a: características de cada zona, integración de la familia, sistema de producción y de gestión.

Las características de la zona fueron relevadas interrogando directamente al productor y a 4 vecinos de los mismos. Se apuntó a la identificación de las instituciones vinculadas con la ganadería trabajando en el lugar. La integración familiar, el sistema de producción (características principales, actividades, calendarios), la conformación del equipo de gestión y el manejo de la información, así como el relacionamiento con técnicos asesores y/o con extensionistas, fueron relevadas también mediante las entrevistas, las recorridas de predio y el análisis de registros existentes.

Cabe aclarar que en este trabajo se suministra la información estrictamente necesaria para el análisis, evitando la identificación de las personas involucradas en los casos estudiados.

\section{RESULTADOS}

\section{Localización del predio-presencia institucional}

La localización de ambos predios, en relación a cercanía con centros poblados y facilidad de acceso es posible de definirla como adecuada y similar para ambos casos. Si bien se ubican en departamentos diferentes, se encuentran respecto a centros urbanos de referencia, en ubicación similar. Los accesos a ambos predios son adecuados, no imponen restricciones más allá de las normales para caminos rurales de balasto.

Respecto a la existencia en la zona de instituciones desarrollando actividades relacionadas con la actividad ganadera, se identificaron en ambas zonas la presencia de las mismas. En el departamento de Rocha se identificó la existencia de tres instituciones, mientras que en el departamento de Cerro Largo fueron dos las identificadas. Se constató la presencia de la 
D.G.D.R. - M.G.A.P., el Instituto Plan Agropecuario y Sociedades de Fomento Rural. En el departamento de Rocha fue identificada además la presencia de un grupo de productores ganaderos organizados, con más de 10 años de actividad, donde participa el caso analizado. Mientras que en el departamento de Cerro Largo, el grupo de productores es de reciente creación, y también participa el caso estudiado.

La identificación de las instituciones que trabajaban en la zona fue realizada por los productores y vecinos. La diferencia mayor entre los casos, en lo relacionado a vinculación con el medio, se observa en la participación a nivel de grupo de productores. El primer caso posee una historia extensa en este tema; mientras que el caso 2, tiene experiencia reciente.

En referencia a la presencia de infraestructura básica en ambas zonas, es posible sostener que no existían diferencias sustanciales entre las mismas (electrificación-telefonía-caminería-servicios de salud-servicios de educación, etc.). Es claro que las características de la zona a pesar de las distancias que las separa, aparecen como muy similares. En el mismo sentido la presencia institucional en las dos zonas favorece el desarrollo de los sistemas bajo estudio.

\section{Integración familiar y equipo de gestión}

La integración familiar y la integración del equipo de gestión se presentan en la Tabla 1 .

Tabla 1. Integración familiar y de equipo de gestión

\begin{tabular}{|c|c|c|c|c|c|c|c|}
\hline Caso & $\begin{array}{c}\text { Integración } \\
\text { familiar }\end{array}$ & Residencia & $\begin{array}{c}\text { Integración } \\
\text { equipo } \\
\text { gestión }\end{array}$ & $\begin{array}{l}\text { Vínculo } \\
\text { familiar }\end{array}$ & Edad & $\begin{array}{c}\text { Nivel } \\
\text { educación } \\
\text { formal } \\
\text { equipo de } \\
\text { gestión }\end{array}$ & $\begin{array}{c}\text { Formación } \\
\text { relacionada a la } \\
\text { ganadería }\end{array}$ \\
\hline 1 & $\begin{array}{c}\text { Matrimonio }+2 \\
\text { hijos }\end{array}$ & $\begin{array}{c}\text { Localidad } \\
\text { cercanaal } \\
\text { predio }\end{array}$ & 2 integrantes & $\begin{array}{l}\text { Productor } \\
\text { y cónyuge }\end{array}$ & $50-49$ & $\begin{array}{c}\text { Primaria } \\
\text { completa } \\
\text { (ambos) }\end{array}$ & $\begin{array}{c}\text { Cursos, giras, } \\
\text { jornadas, charlas }\end{array}$ \\
\hline 2 & $\underset{\text { hijo }}{\text { Matrimonio }}+1$ & $\begin{array}{c}\text { Compartida } \\
\text { predio- } \\
\text { localidad } \\
\text { cercana al } \\
\text { predio }\end{array}$ & 1 integrante & Productor & 49 & $\begin{array}{c}\text { UTU } \\
\text { completa }\end{array}$ & Jornadas, charlas \\
\hline
\end{tabular}

La integración de la familia era similar en ambos casos. Ambas familias se integraban por el titular y cónyuge, la diferencia radicaba en que en el caso 1, posee 2 hijos y en el caso 2, posee 1 hijo. Desde el punto de vista de la gestión del predio, esta diferencia no era relevante, dado que los hijos no participaban del equipo de gestión en ningún caso. En este sentido se pudo observar que el equipo de gestión estaba formado por el matrimonio en el caso 1 y por el productor exclusivamente en el caso 2. La participación de la familia en las decisiones productivas es una de las fortalezas de estos sistemas de producción familiares.

Respecto a la residencia, en el caso 1 residían en una localidad cercana. En el caso 2, el productor compartía residencia entre el predio y localidad cercana; mientras que el resto del núcleo familiar residía la mayor parte del tiempo en localidad cercana.

La edad de los gestores de los predios, oscilaba los 50 años. Por su parte los niveles de educación formal fueron de educación primaria para los 2 integrantes del equipo para el caso 1 ; mientras que para el caso 2 , fue de UTU completa (bachillerato tecnológico). En lo relacionado a la formación relacionada con ganadería, el caso 1 tenía una intensa actividad de formación “profesional”. Para el caso 2, la formación era concurrir a charlas relacionadas con la ganadería.

Respecto a las finalidades de los sistemas familiaexplotación estudiados, surgen de las entrevistas las mismas. Las finalidades si bien son diferentes entre ambos sistemas, son del estilo de: mantener vida rural, asegurar crecimiento de hijos, generar un mayor nivel de ingreso familiar. Las mismas fueron validadas por 
las familias involucradas. En base a esas finalidades, se plantean las distintas estrategias para llevar adelante la vida familiar y el sistema de producción.

\section{Sistema de producción}

En cuanto al sistema de producción, ambos casos llevaban adelante sistemas de cría en bovinos, sobre campos de sierra, en el Este de Uruguay. Los objetivos que dijeron tener los productores y familias eran similares; la generación de más ingresos era el objetivo principal declarado, a partir de la producción de terneros. No obstante, ambos sistemas productivos presentaban diferencias en aspectos centrales de los mismos. La disponibilidad de infraestructura predial, para ambos casos era similar. Se contaba con instalaciones básicas para el manejo del ganado y empotreramiento similar. El caso 1, luego de haber tenido un sistema mixto de vacunos y lanares, poseía únicamente vacunos; mientras que el caso 2 , tenía cría lanar además de vacunos.

En lo relacionado a las prácticas de manejo animal, se observó en el caso 1, destacados niveles de intervención en diferentes etapas del proceso criador. El manejo del rodeo estaba basado en la utilización de una serie de conocimientos aplicados, más comúnmente denominadas tecnologías de proceso. Desde la clasificación por estado fisiológico y condición corporal de las vacas de cría; pasando por la planificación del período de entore y de destete; hasta el control temporal del amamantamiento; utilizados de manera estructuralsistemática anualmente. En base a lo observado fue posible definir la existencia de elevados niveles de intervención-control de lo que sucedía dentro del predio en relación con los animales. De acuerdo a lo observado la intervención además de la aplicación de las tecnologías de proceso, era la utilización de tecnologías de insumos.

La suplementación estratégica, de categorías de reemplazo, era una estrategia definida como estructural en el caso 1. Esta práctica contribuía al objetivo de entorar las vaquillonas con 2 años de edad y 280-290 kilos/peso vivo por cabeza.

Por su parte el caso 2, realizaba ciertas prácticas de manejo del rodeo de cría, aunque con menor rigurosidad. Las mismas no eran un componente estructural del sistema como en el caso 1; sino que dependía su uso de las condiciones del año y de la percepción que tenía el productor-decisor. La determinación de los períodos de entore y de destete, eran flexibles y dependían de como venía el año. En años que se los definía como "malos", el toro permanecía con el rodeo de cría durante gran parte del año. Mientras que en esos años malos los destetes se realizaban de acuerdo al estado corporal y peso de los terneros, y no en relación a una fecha pre-establecida. La clasificación por estado fisiológico y condición corporal se realizaba sin apoyo de herramientas de diagnóstico objetivas. El uso del control de amamantamiento no era estructuralmente utilizado, debido al efecto negativo que tenía sobre el estado de los terneros según opinión del productor. La utilización de tecnologías de insumo, como el uso de la suplementación, era exclusivamente utilizada para salvataje de animales frente a situaciones de crisis forrajeras.

Respecto al componente vegetal del sistema de producción, se pudo observar y cuantificar que la base era el campo natural, con un porcentaje de mejoramientos forrajeros del orden del 15\% del área, para el caso 1 . Mientras que para el caso 2, también la base era el campo natural, con mejoramientos forrajeros en el $10 \%$ del área. También en las prácticas de manejo vegetal se observaron diferencias.

El caso 1 realizaba la utilización de los mejoramientos forrajeros en base a subdivisiones con alambrado eléctrico, realizando una muy buena utilización, con períodos de descanso. La utilización del área mejorada era planificada como estratégica y estaba reservada para la parición del rodeo y para una etapa en la vida de la recría. La refertilización anual de los mejoramientos era una decisión estratégica y una práctica prioritaria y anual común. Por su parte en el caso 2, si bien se pretendía realizar una utilización estratégica, el estado de los animales no lo permitía. Las áreas de mejoramientos forrajeros, en gran proporción eran utilizadas con animales con deterioro de la condición corporal. Las 
refertilizaciones se realizaban de acuerdo a la liquidez financiera, no era una práctica considerada como prioritaria.

Los suelos sobre los que estaban ubicados los predios de acuerdo al índice Coneat promedio eran similares y se los podría caracterizar en referencia a su aptitud como medio-bajos. El valor promedio era 65.

Por el lado del campo natural, si bien no eran iguales; las diferencias mayores se observaron en lo relacionado a la disponibilidad de forraje (altura de pasto), con la que se los manejaba en los dos casos. En el caso 1, la disponibilidad era superior y los animales entraban a los potreros con alturas de forraje superiores que el caso 2, en el orden del $60 \%$ superior. La entrada a los potreros era en general con alturas de forraje superiores a $\operatorname{los} 8 \mathrm{~cm}$.

En relación a la disponibilidad de pasturas y haciendo referencia a la dotación animal con la que se observó que trabajaban los casos estudiados, se identificaron diferencias de importancia. El caso 1 trabajaba sistemáticamente y desde hace más de 10 años con dotaciones ajustadas a la oferta de forraje del sistema. En valores de dotación (promedio) en unidades ganaderas/hectárea, esto significa en el orden de las 0,75 UG/ha. El ajuste de la carga animal del sistema, en este caso 1, completa un escenario de fuertes niveles de intervención en busca del objetivo manifestado.

Por su parte el caso 2, de acuerdo a lo observado en las visitas y a los registros disponibles, la dotación animal promedio oscilaba 1,0 UG/ha. Este productor también realizaba ajustes de carga a la entrada del invierno. El ajuste que se realizaba, permitía mitigar los problemas en el invierno, aunque no permitía trabajar con adecuados volúmenes disponibles de forraje.

El estado de situación del proceso de producción, con niveles de intervención y ajuste importantes que se observaron en el caso 1, era producto de un tomador de decisiones con gran capacidad de gestiónintervención.

Los niveles de intervención desarrollados le permitieron al caso 1, cosechar a lo largo de los últimos 8 años elevados y estables indicadores productivos. La tasa de marcación promediaba el 94\%; mientras que los kilos de carne producidos/hectárea alcanzaron valores del orden de los 110-120 kilos. Por su parte los niveles de ingreso familiar/hectárea promedian en los últimos años los 140-150 dólares, resultado realmente destacado.

Mientras tanto en el caso 2, los menores niveles de intervención, se reflejaban en menores indicadores tanto productivos como reproductivos. La tasa de marcación alcanzaba valores $60 \%$ promedio, y en los años mejores máximos de $67 \%$. Por su parte los kilos producidos promediaban los 60 kilos/hectárea. Mientras el ingreso familiar/hectárea promediaba los 60 dólares.

\section{Sistema de gestión}

En la Tabla 1 se muestran los integrantes del equipo de gestión. En el caso 1 el equipo estaba conformado por el productor y cónyuge; mientras que en el caso 2, el equipo era unipersonal, integrado por el productor.

Respecto a la diferenciación en actividades de campo y de gestión propiamente, en el caso 1 se identificó una división para las actividades que requieren de esfuerzo físico, como las relacionadas con los animales directamente. Estas actividades las realizaba el productor titular; mientras que las actividades más relacionadas con la gestión eran desempeñadas por ambos titulares. Para el caso 2, equipo unipersonal, era el productor quien realizaba la totalidad de las actividades tanto físicas como de gestión del sistema.

\section{La generación y el uso de la información generada en el predio}

Entendiendo actividades de gestión, como aquellas que comienzan con la captura de los datos que se generan en los predios y continúan con otras más relacionadas al control, análisis, planificación y toma de decisiones, se encontraron diferencias.

El caso 1, el manejo de la información está planteado como un sistema, dónde los componentes tenían su responsable claramente definido. La captura de datos se realizaba en libreta de campo y/o con boletas/comprobantes de gastos archivadas en una carpeta. La responsabilidad sobre los datos de campo, era del 
productor; mientras que la responsabilidad de otros datos era del integrante del equipo que realizaba la transacción. La registración se realizaba en una planilla electrónica diseñada por el hijo del productor; con una periodicidad semanal; por parte del titular o cónyuge. Respecto al uso de la información generada, se identificó un nivel importante de utilización de la misma. En relación a aspectos del sistema de producción el uso estaba relacionado al análisis de los resultados físicos y la contrastación contra lo planificado. Mientras que en referencia a aspectos económicosfinancieros, el análisis de los costos de producción, el precio de venta logrado y el flujo y saldo de caja, eran los centros de interés. Se observó también uso de la información para planificar el año siguiente. La planificación focalizaba en resultados físicos, precios posibles por la venta de productos y evolución esperable de costos de producción. La misma se realizaba de forma escrita. La experiencia en estas actividades tenía 10 años. Para esta familia, las actividades de manejo de información, eran consideradas de gran importancia; en nivel similar a las actividades de campo. El caso 2, la situación era algo diferente. Respecto a datos productivos, los mismos se capturaban en libretas de campo y/o almanaques. Mientras que para los datos de otro origen, se guardaban boletas u otro tipo de comprobantes, en forma no rigurosa. No se identificó una rutina de asiento de los datos en ningún soporte por parte del productor. La misma se desarrollaba cuando el técnico extensionista lo visitaba, ocasión en la cual registraban los datos en la herramienta denominada Carpeta Verde.

El uso de la información generada, se identificó como marginal y se vinculaba a aspectos relacionados con el control de movimientos del stock de animales y en el precio logrado por cabeza vendida. Las actividades de planificación estaban relacionadas con la alimentación animal. La experiencia en este tipo de actividades era reciente, 3 años. Las actividades de manejo de información, eran secundarias.

Información extra predio: papel del asesoramiento técnico y extensión
Respecto a la información extra predio, su fuente y utilización, se observaron también diferencias entre los casos estudiados. El caso 1, la fuente de información extra predio definida como de mayor relevancia en relación a aspectos técnicos fueron las discusiones en el ámbito del grupo de productores, el intercambio con profesionales del área agraria (Ingenieros Agrónomos y/o Veterinarios), seguida por orden de importancia por jornadas-charlas técnicas y luego revistas de contenidos técnicos. Con referencia a información relacionada con precios de venta y pronósticos meteorológicos, internet y la radio fueron las mencionadas como de mayor relevancia. La experiencia personal anterior si bien es de importancia, fue relativizada, debido a la disponibilidad y uso de nuevos conocimientos disponibles.

El caso 2, definió como principal fuente de información la experiencia personal anterior. En segundo lugar fue mencionado el intercambio con otros productores-vecinos y esporádicamente técnicos del área agraria de su conocimiento. La radio de la localidad fue la fuente de información por excelencia en lo relacionado a precios y pronósticos meteorológicos. Fue mencionado también como fuente de importancia el técnico extensionista que lo visita frecuentemente.

Ambos casos difieren entonces en el papel que otorgan al asesoramiento técnico y extensión. En el primer caso hay una valorización del aporte de la información técnica aportada por el técnico directa (charlas, revistas, comunicación personal) o indirectamente (grupos). La metodología grupal es desde siempre una estrategia recomendada para el trabajo con productores no solo por su eficacia en generar cambios a través de la influencia que tiene los pares en la toma de decisiones sino también por la eficiencia que representa poder compartir el costo del asesoramiento técnico. En el caso de las políticas públicas que han privilegiado esta metodología para el trabajo con productores el costo es subsidiado. Lamentablemente la mayoría de los grupos formados al amparo de este subsidio desaparecen cuando los proyectos terminan; los casos exitosos se relacionan con grupos que ya existían antes 
del proyecto y se habían formado de manera genuina.

\section{Toma de decisiones y estilos de gestión}

La toma de decisiones relacionadas con aspectos productivos de importancia como es la dotación animal, en el caso 1 se realizaba observando en forma permanente el estado de las pasturas. Las decisiones de ajuste de carga, de cambio de potrero, de eventuales medidas de suplementación eran tomadas en base a la disponibilidad de pasto de cada potrero.

En el caso 2 este tipo de decisiones, si bien tenía un componente de observación del estado de las pasturas, el componente de mayor importancia para la toma de las decisiones era el estado corporal de los animales. Cabe mencionar en relación a este aspecto de estilo de gestión, que los objetivos productivos en el caso 1 estaban claramente definidos y eran respetados completamente. La producción de terneros con elevados pesos al destete (175 kilos/cabeza) era el objetivo definido claramente. Las decisiones de cualquier tipo, eran subordinadas al mencionado objetivo. Respecto al caso 2, si bien el objetivo productivo manifestado era la producción de terneros, en oportunidades ese objetivo era desplazado por el engorde de vacas de reemplazo. El peso promedio de los terneros al destete era de $130 \mathrm{kilos} /$ cabeza.

Respecto a la incertidumbre, los factores generadores en estos productores ganaderos, son por un lado los climáticos (sequías principalmente) y las variaciones de precios de los productos. Cada productor lleva adelante diferentes estrategias para mitigar los efectos de los mismos.

En el caso 1, la dotación animal ajustada a la oferta forrajera fue la estrategia identificada como más importante en relación a los factores climáticos. Esta estrategia le ha permitido aún en años de ocurrencia de importantes eventos de sequía (2005-2006 y 20082009), lograr elevados rendimientos reproductivos y productivos (75\% de marcación - 80 kilos de carne producidos/hectárea). Respecto a las variaciones de precios, las estrategias identificadas fueron dos. La primera asociada a la estrategia anterior, un muy ajustado sistema productivo le permite elevados niveles de productividad y productos de alta calidad (terneros pesados y parejos; vacas de descarte de 400 kilos promedio/cabeza) para enfrentar la caída de precios. La segunda asociada al control de costos de producción. El control de costos productivos no sólo por unidad de superficie, sino fundamentalmente por unidad de producto es fundamental. Por último, una estrategia general, que se la definió como de "manejo seguro", es la disponibilidad de determinados niveles de liquidez (Tabla 2).

Tabla 2. Características de diferentes estilos de gestión

\begin{tabular}{|c|c|c|}
\hline & Productor 1 "analítico -pastoril" & Productor 2 "experiente -ganadero" \\
\hline Equipo de gestión & Pareja; división de tareas & Unipersonal \\
\hline Uso de tecnología & $\begin{array}{c}\text { Intenso uso de tecnologías de } \\
\text { proceso, medio alto uso tecnologías } \\
\text { de insumos }\end{array}$ & $\begin{array}{l}\text { Medio bajo uso de tecnologías de } \\
\text { proceso, }\end{array}$ \\
\hline Control & $\begin{array}{c}\text { Estado pasturas; Condición corporal } \\
\text { animales; Control amamantamiento; } \\
\text { Diagnósticos actividad ovárica y } \\
\text { gestación }\end{array}$ & $\begin{array}{l}\text { Condición corporal animales; Control } \\
\text { amamantamiento }\end{array}$ \\
\hline Planificación & $\begin{array}{l}\text { Entore; destete; Suplementación } \\
\text { invernal; Pastoreo controlado en } \\
\text { franjas; Descanso pasturas; } \\
\text { Refertilizaciones anuales; Altura de } \\
\text { pastura para su utilización; Manejo } \\
\text { carga animal ajustado a pasturas } \\
(0,74-0,77 \text { UG/ha) }\end{array}$ & $\begin{array}{l}\text { Entore flexible en duración; destete } \\
\text { flexible según estado terneros; } \\
\text { Suplementación de emergencia; } \\
\text { Carga animal elevado }(1,0-1,2 \\
\text { UG/ha) }\end{array}$ \\
\hline $\begin{array}{l}\text { Decisiones inversión- } \\
\text { reinversión }\end{array}$ & Anuales; planificadas; prioritarias & Eventuales; no prioritarias \\
\hline
\end{tabular}


En el caso 2, la venta de animales como medida del ajuste rápido de la dotación fue la estrategia identificada para enfrentar condiciones climáticas adversas. Esta estrategia operaba una vez que la observación del estado corporal de los animales manifestaba un pro- blema. Respecto a las variaciones de precios, la estrategia identificada fue la reducción de costos de producción y de retiros familiares. La disponibilidad de determinados niveles de liquidez, no se veía como posible de efectivizar normalmente (Tabla 3 ).

Tabla 3. Gestión de la información según estilo de gestión

\begin{tabular}{|c|c|c|}
\hline & Productor 1 "analítico -pastoril" & Productor 2"experiente -ganadero" \\
\hline $\begin{array}{l}\text { Manejo de información } \\
\text { intra-predial }\end{array}$ & $\begin{array}{l}\text { Captura de datos definida; elevado uso de } \\
\text { la información; controly análisis de costos } \\
\text { y precios; saldo de caja; planificación del } \\
\text { próximo ejercicio; manejo de distintos } \\
\text { escenarios }\end{array}$ & $\begin{array}{l}\text { Informalidad en captura de datos; bajo } \\
\text { uso de la información; control de stock; } \\
\text { control y análisis precios venta }\end{array}$ \\
\hline $\begin{array}{l}\text { Fuentes de información } \\
\text { externas (ATER) }\end{array}$ & $\begin{array}{l}\text { Grupo de discusión; charlas - jornadas } \\
\text { técnicas; revistas técnicas; internet } \\
\text { (precios, pronósticos meteorológicos) }\end{array}$ & $\begin{array}{c}\text { Experiencia personal; radio local } \\
\text { (precios, pronósticos meteorológicos) }\end{array}$ \\
\hline Central de control & Estado de las pasturas & Estado de los animales \\
\hline $\begin{array}{c}\text { Objetivos productivos } \\
\text { del sistema }\end{array}$ & Claros; bien definidos; permanentes & Claros; variables \\
\hline \multirow{3}{*}{$\begin{array}{l}\text { Manejo de riesgo } \\
\text { climático y de mercado }\end{array}$} & Dotación ajustada a la oferta de forraje & Ajuste de la dotación por ventas \\
\hline & Productos de excelente calidad & $\begin{array}{l}\text { Reducción de costos productivos y } \\
\text { retiros familiares }\end{array}$ \\
\hline & Control de costos por kilo de producto & \\
\hline
\end{tabular}

En relación a las decisiones de inversión, en ambos casos las mismas estaban condicionadas al saldo de caja. La disponibilidad de efectivo era considerada la primera restricción posible, dado que la toma de créditos no era una opción considerada en ninguno de los 2 casos.

En el caso 1, las inversiones relacionadas al mantenimiento de los mejoramientos forrajeros tenían prioridad sobre otras inversiones posibles y estaban planificadas anualmente. Mientras que en el caso 2, las inversiones productivas eran escasas y no se planificaban, sino que dependían de la oportunidad. En este caso las inversiones-gastos relacionados con la familia tenían prioridad.

En las Tablas $\mathrm{N}^{\circ} 2$ y $\mathrm{N}^{\circ} 3$ se sintetizan aspectos más relevantes que caracterizan los estilos de gestión.

\section{DISCUSIÓN}

Piñeiro et al. (1998) construye una tipología de productores lecheros, a partir de diferentes comportamientos de los mismos respecto a cómo realizan la gestión del predio. En primer lugar caracteriza los distintos comportamientos, identificando las principa- les variables que inciden en dicho comportamiento diferencial. Se agrupa los productores según la forma de llevar o no, registros físicos y registros económicos. Se construye una tipología de productores, distinguiendo cuatro categorías de productores. Se señalan 7 factores que inciden en la pertenencia a estos grupos (escala, composición familiar, nivel y participación en instancias de capacitación, uso de tecnologías, pertenencia a grupos, vinculación con el mundo técnico). Los últimos tres factores se vinculan con el asesoramiento técnico y la extensión.

Los productores definidos como tipo 1, no utilizan tecnologías recomendadas, llevan registros físicos de manera muy elemental, presentan dificultades para comprender operaciones complejas que implican los cálculos económicos, no participan en instancias de capacitación y la presencia técnica en sus sistemas de producción es insignificante. Por su parte los productores tipo 2, utilizan alguna de las tecnologías recomendadas, los registros físicos son llevados en soportes más elaborados, los niveles de capacitación son superiores, la relación con los técnicos es algo más fuerte y la integración familiar con el predio como 
centro, es abastecedora de mano de obra. Mientras los productores tipo 3 , presentan un nivel de capacitación superior y además participan de instancias de capacitación, hacen un uso importante de las tecnologías recomendadas, tanto llevan ordenadamente registros físicos como económico-financiero, los que además de para controlar utilizan para planificar el predio. La familia está integrada al predio, aportando mano de obra, incluso en oportunidades en exceso. El último tipo, el tipo 4, se caracteriza por un uso intenso de las tecnologías recomendadas, el uso también intenso de la información para las decisiones tanto productivas como económico-financieras y en la planificación a mediano plazo. La participación de la familia en la gestión del predio es de importancia. El relacionamiento con la asistencia técnica o servicios de extensión es frecuente e intenso y presentan en general buenos a muy buenos resultados productivos y económi$\cos$.

Haciendo referencia a nuestro trabajo, el estilo de gestión identificado como "experiente-ganadero"; tiene similitudes en sus características principales, con el productor tipo 2, identificado por Piñeiro et al. (1998). Es un estilo que se caracteriza por el uso de algunas o varias técnicas productivas "recomendadas". Los datos básicos relacionados a los aspectos físico-productivos del sistema de producción son los que utiliza. Los mismos se registran en soportes sencillos. Respecto al nivel de educación formal, el mismo no sería una restricción, dado que cuentan con un nivel equivalente a enseñanza secundaria. Una de las preocupaciones más importante del sistema es el control del estado de los animales. Respecto al ciclo familiar, es una familia consolidada con hijo en etapa adolescente. Presentando un excedente de mano de obra familiar disponible y una desfavorable relación entre “productores" y “consumidores" en el ámbito familiar. Por su parte la presencia o cercanía con la asistencia técnica o extensionistas, existe pero es restringida. Mientras tanto, si se analiza el estilo de gestión "analítico pastoril"; las similitudes con lo identificado por Piñeiro et al. (1998) se presentan con el tipo 4, deno- minado "empresario diversificado". Este tipo de productor presenta un estilo de gestión basado en el uso prácticamente completo de las tecnologías disponibles. Respecto a la registración de datos, es también completa y en soportes más o menos sencillos. El uso de la información generada es intenso, en control y en planificación del predio. La generación de indicadores, el uso de los mismos para ejercer la función de control y de evaluación, como también la de planificación es de destaque. Los resultados globales del predio, son generalmente definidos como buenos a muy buenos. La vinculación con el mundo técnico es alta, y se da a partir tanto de consultas, como de la participación en grupos de productores y en jornadas técnicas. Los casos estudiados, contaban con recursos naturales similares (área-tipo de suelo-tipo de campo natural); con infraestructura similar (instalacionesmejoramientos forrajeros- subdivisiones); no obstante los resultados productivos-económicos logrados eran sustancialmente diferentes. Por lo antes dicho surgen evidencias para este contraste de casos, que los recursos naturales disponibles por los sistemas estudiados no son absolutamente determinantes en las diferencias de resultados productivos y económicos logrados. Los niveles de capacitación en temáticas relacionadas a ganadería eran similares para ambos casos de productores. La generación y uso de información relacionada al sistema de producción presentaba diferencias de importancia entre los casos estudiados. Mientras que en el caso 1, que lograba resultados superiores, la generación de información interna a la explotación se realizaba en forma sistemática y su utilización se centraba en control y planificación, como señala Piñeiro et al. para el productor tipo 4. En el caso 2, con resultados inferiores, la generación de información interna era algo secundario, y su uso era principalmente para controles físicos y de stocks, similar situación al productor tipo 2 de Piñeiro et al. (1998).

Mientras tanto el papel del asesoramiento técnico y la extensión a través de las fuentes de información externas utilizadas por los casos estudiados, presentaban diferencias. En el caso 1 se mencionaba las discusio- 
nes en el ámbito del grupo de productores como la fuente de mayor importancia jerarquizando el aporte de la información técnica y el relacionamiento con técnicos asesores y extensionistas, como define Piñeiro et al. (1998) para el productor tipo 4. Para el caso 2 se destacaba la experiencia personal como el factor relevante para la toma de decisiones, mientras que la vinculación con el "mundo técnico", era escasa; característica similar al productor definido como tipo 2 por Piñeiro et al. (1998).

\section{CONCLUSIONES}

Es posible de señalarse que el marco teórico elegido como la propuesta metodológica implementada permitió caracterizar adecuadamente los 2 sistemas familia-explotación.

A partir de la misma se ha avanzado en el conocimiento de factores que determinan diferencias en resultados productivos-económicos a escala de predio criador familiar y en la identificación de dos estilos de gestión bien diferenciados. Una apropiada utilización del conocimiento disponible, de tecnologías de proceso y de insumos, a pesar de contar con recursos naturales con ciertas limitaciones (Índice Coneat promedio suelos $=65)$, permiten el logro de niveles elevados y sustentables de producción y de ingresos familiares. Un estilo de gestión definido como "analíticopastoril", asociado a la observación del estado de las pasturas para tomar decisiones; donde las actividades de gestión tienen un lugar destacado dentro de las actividades del predio; con niveles de intervención por parte del decisor de importancia en los procesos clave del sistema productivo; estaba en uno de los casos estudiados directamente asociado al logro de elevados resultados prediales, tanto en lo productivo como en lo económico.

Por su parte el papel de ATER es relevante en los resultados obtenidos, a través de la información tecnica y del trabajo en grupos. La información técnica se relaciona con el conocimiento explicito mientras que los grupos representan un ambiente adecuado para interactuar con diferentes tipos de conocimiento. En efecto, el grupo permite identificar (recuperar) el conocimiento tácito, cuando se comparten experiencias (conocimientos tácitos), o permite también compartir informaciones (conocimientos explícitos) que al interiorizarse en las personas se transforman en conocimiento tácito. Indudablemente que el técnico al moderar en el grupo va trasformando conocimiento tácito en explícito (informaciones) y por otra parte debe vigilar que las interacciones entre diferentes conocimientos no generen como resultado confusión. En definitiva, una adecuada interacción entre diferentes conocimientos genera oportunidades de aprendizaje que explican los resultados

Surge evidencia que factores relacionados al gestor y equipo, como ser estilo de gestión "analítico-pastoril", elevados niveles de intervención en los procesos de producción, intensa utilización de conocimientos disponibles relacionados con el manejo de animales y de pasturas (campo natural-mejoramientos forrajeros), intensa utilización de tecnologías de proceso, definición clara de objetivos productivos, mantenimiento de los mismos a pesar de la aparición de "negocios" coyunturales atractivos (del estilo del engorde de vacas de reemplazo), definiciones claras y sólidas en relación a decisiones de inversión-reinversión, estaba en uno de los casos estudiados directamente relacionados al logro de destacados y sustentables resultados productivos-económicos; a pesar de recursos naturales de limitada productividad potencial. Mientras que el otro estilo de gestión denominado "experienteganadero"; con menores niveles de intervención y en términos generales un menor uso de las tecnologías disponibles, mostraba resultados sistemáticamente inferiores.

El contraste presentado entre dos modalidades de implementar el funcionamiento de sistemas ganaderos criadores con una marcada diferencia en sus resultados económico-productivos y el establecimiento de una serie de hipótesis sobre algunos de los determinantes representados en los estilos de gestión descriptos sugiere la necesidad de desarrollar estudios complementarios. En el mismo sentido una de las líneas de 
trabajo deberá apuntar a mejorar nuestra comprensión sobre los componentes de los estilos de gestión, en términos de experiencias de vida-factores humanosdinámicas sociales. Al mismo tiempo deberá avanzarse en la comprensión de cómo es posible desarrollarpotenciar estos comportamientos. Finalmente los aspectos vinculados con el trabajo en grupos deberán estudiarse desde una perspectiva que priorice el uso de esta metodología en una escala mayor de público objetivo, al tiempo que es importante estudiar los factores que aseguran la sostenibilidad de los grupos desde su formación.

\section{REFERENCIAS BIBLIOGRÁFICAS}

Alvarez, J. 2005. Notas sobre la naturaleza del proceso decisorio que realizan los productores agropecuarios en tanto gestores de sus explotaciones: Curso de Sistemas de Información para Empresas Agropecuarias, Montevideo (Urugay): Maestría en Ciencias Sociales. Facultad de Agronomía.

Bonnemarie, J., J. P. Deffontaines y P. L. Osty. 1980. “Observations sur l'agriculture en zones défavorisées à partir de recherches sur le fonctionnement des explotationsagricoles." C.R. Acad. Agri. Fr. 66 (4): 361-375

Bonneviale, J. R., R. Jussiau y E. Marshall. 1989. Approche Globale de L'exploitation Agricole. Dijon(Francia): INRAP-FOUCHER.

Beranger, C. y B. Vissac. 1994. “An holistic approach to livestock farming systems: theoretical and methodological aspect". En: The study of livestock farming systems in a research and framework. Gibon, A. y J. C. Flamant (eds.). Wageningen (Holanda): Wageningen Press.

De Hegedus, P. y H. Morales. 1996. ”Algunas consideraciones sobre el enfoque sistémico y su importancia para la extensión rural." Extensão Rural (3): 49-60

Dillon, J. L. 1976. "The economics of systems research" Agricultural Systems 1: 15-22

Duru, M. 1980. Exploitation agricole et analyse de systeme: Mise au point méthodologique. Paris (Francia): INRA-SAD.

Figari, M. 2008. Resultado económico y subjetividad: representaciones sociales de productores familiares de Paysandú, Tesis de Maestría, Universidad de la República de Uruguay. Montevideo(Uruguay).

Marshall, E., J. R. Bonneviale y I. Francfort. 1994. Fonctionnement et diagnostic global de l'exploitation agricole. Une méthode interdisciplinaire pour la formation et le développement. Dijon (Francia): ENESAD-SED.

Osty, P. L. 1978. "L'exploitation agricole vue comme un systéme. Diffusion de l'innovation et contribution au développement." Bulletin Technique d'Informations (326): 43-49

Patton, M. Q. 1980. Qualitative evaluation methods. Beverly Hills (EEUU): Sage Pub., Inc.

Pedemonte, A., C. Molina, J. Álvarez. 2014. "Proceso de toma de decisions relacionado al uso del suelo de explotaciones hortícolas familiares." Agrociencia Uruguay 18(1): 41-52

Piñeiro, D., M. Chiappe, y F. Graña F. 1988. La gestión en establecimientos lecheros: una tipología de los productores según su disposición al uso de registros físicos y económicos. Agrociencia Uruguay 2(1): 125-133

Ruíz, R. y Oregui L. 2001. “El enfoque sistémico en el análisis de la producción animal: revisión bibliográfica." Invest. Agr.: Prod. Sanid. Anim. 16(1): 29-61

Wilson, K. y G. E. B. Morren. 1990. Systems approaches for improvement in agriculture and resource management. New York (EEUU): Mac Millan Pub.

Yin, R. K. 1989. The case study research: Design and methods. Newbury Parks (EEUU): Sage Publications. 